\title{
Design and Implementation of Smart Ocean Visualization System Based on Extended Reality Technology
}

\author{
Xu Han ${ }^{1}$, Jingming Liu ${ }^{1}$, Baohua Tan ${ }^{2, *}$ and Lucheng Duan ${ }^{2}$ \\ ${ }^{1}$ School of Industrial Design, Hubei University of Technology, Wuhan 430068, \\ China \\ ${ }^{2}$ School of Science, Hubei University of Technology, Wuhan 430068, China \\ E-mail:tan_bh@126.com \\ ${ }^{*}$ Corresponding Author
}

Received 11 December 2020; Accepted 24 December 2020;

Publication 09 March 2021

\begin{abstract}
In the context of building a maritime power, building a smart ocean is one of the important means to promote ocean development. However, there is currently a lack of effective smart solutions for ocean development to integrate and manage ocean information. To solve the problem of insufficient development of smart ocean systems, a smart ocean visualization app based on extended reality technology has been developed, using Python crawler technology to collect ocean big data, and produce the system through Unity software. The app is built using C\# programming, and the AR animation on the app is realized with the AR Foundation plug-in. Through terminals such as mobile phones or computers, it provides users with real-time ocean data query and expanded realistic ocean tourism services. The visualization function of the system realizes innovation in the way of querying marine data and makes up for the lack of development of smart marine apps.
\end{abstract}

Keywords: Smart ocean, extended reality technology, Unity3D, visual interaction.

Journal of Web Engineering, Vol. 20_2, 557-574.

doi: 10.13052/jwe1540-9589.20215

(C) 2021 River Publishers 


\section{Introduction}

The development of smart ocean should be based on high-tech such as ocean integrated stereoscopic perception, Internet real-time information transmission, large data, cloud computing and knowledge mining, with information infrastructure such as ocean integrated perception network, marine information communication network and ocean large data cloud platform as the main body, build the intelligent application service group of marine information, and establish standard quality, operation and operation services, technical equipment and information security system throughout the links. At present, ocean big data has the characteristics of multi-source, polymorphism, and diversity. There are problems of low data processing efficiency, insufficient utilization of marine information resources, and more static forms of complex marine environment information expression, but less dynamic presentation. Meanwhile, the Corona Virus Disease 2019 (COVID-19) is spreading rapidly worldwide, vessel quarantines and tie ups threaten to disrupt raw material supply, as do incidents of contagion in seafood processing plants (Sapin and Cherry 2020): temporary shut-downs and volume reductions are being implemented in accordance with social distancing requirements. Many countries have taken measures to restrict the import of seafood, and some imported aquatic products also have the risk of COVID-19 infection, which brings about the problem that the source information of seafood is not clear, and eating seafood has the risk of infection. At the same time, due to the infectivity and diffusion of the virus, the latest research report of the world travel and tourism council (WTTC) lists up to 75 million workers at immediate job risk as a result of COVID-19. Research reveals a potential Travel Tourism GDP loss in 2020 of up to US\$2.1 trillion, the use of XR technology to help the marine tourism industry turn from "crisis" to "opportunity" is also an important point in promoting this study.

The information fragmentation of ocean big data, the opaque source of seafood in the catering industry, and the response of the tourism industry to the epidemic crisis have made XR technology to assist the physical tourism industry a rigid demand. In view of these problems, this research has led to the development of a smart ocean visualization system based on the extended reality technology by using the popularization of 5G network technology and XR technology. In the general direction, it helps the government to conduct comprehensive integrated visualization management of closed and fragmented marine data, and help marine researchers obtain marine information data more intuitively, and also provides solutions for the import and 
export trade of marine products and marine tourism under the influence of the epidemic situation. In the system, the source and virus pollution information of the imported seafood are updated and displayed in real time, so that both businesses and consumers can buy at ease; AR dynamic visualization display of marine life contained in the system, VR display of Island Scenic Spots in the ocean, and annotation of tourism routes in the system can help the public understand marine life and marine ecosystem, enhance the public's interest in marine tourism, and promote the development of marine tourism.

\section{Introduction to Research Projects}

Extended Reality (XR) is a general term that includes Augmented Reality (AR), Virtual Reality (VR), and Mixed Reality (MR). Augmented reality (AR) technology is used to enhance a user's perception and interaction with the natural world through the superimposition of digital information on physical images. Virtual reality (VR) is a high-level computer-based interactive and multimedia technology that offers virtual objects and environments that represent actual environments. This immersion is the measure of users' level of "real-ness" in the electronically simulated environment. Virtual reality (VR) technology has three basic characteristics, namely, scene, interaction and space. Therefore, the map combined with virtual reality technology will have the following features: (1) the virtual reality map meets the three basic characteristics: measurable, intuitive and at-a-glance; (2) Virtual reality map enables users to immerse multi body experience environment (simulation); (3) the virtual reality map is "accessible" and interactive.

Therefore, the map combined with virtual reality technology will have the following features: (1) the virtual reality map meets the three basic characteristics: measurable, intuitive and at-a-glance; (2) Virtual reality map enables users to immerse multi body experience environment (simulation); (3) the virtual reality map is "accessible" and interactive. Based on these characteristics, virtual reality map has incomparable advantages in map expression. Combining the AR, VR, and MR included in XR with the smart ocean visualization system, and realize the dynamic integrated visualization of ocean information based on the collected ocean data. This research combines the AR, VR, MR included in XR with map navigation to create a smart ocean visualization system. Based on the collected ocean data, including ocean remote sensing data, ocean water temperature data, ocean meteorological data, ocean chemical data, and marine life data and other types of data, it can realize the dynamic integrated visual presentation of marine information. 
In this study, unity3d software is used to complete the design and development of the smart ocean extended reality visualization system. Through the tour in smart ocean virtual reality system, along with the AR display of marine life and marine products on the mobile terminal, the changes of ocean data can be observed in real time, and the ocean scene roaming and real-time ocean data query services can be provided for users, it makes the complex marine environment information express intuitively and dynamically. It enables the government to make decisions and marine researchers obtain marine data and information when conducting scientific research more conveniently, enables the seafood traders can see the source of marine products clearly, and also allows the public to have a more intuitively understanding of marine ecosystem and marine life habits, so as to enhance the attractiveness of marine tourism to the public.

\section{Marine Data Collection and Model Generation}

\subsection{Project Preparation}

(1) Overall planning. According to the general idea of top-level design, the project uses the method of system theory to make overall planning for all aspects, levels and elements of large-scale complex system construction from the overall perspective, so as to centralize effective resources and achieve the objectives of structural optimization, functional coordination and resource integration. First, make a good overall plan for the development of the smart ocean virtual reality system, determine the specific research framework and detailed research ideas of the project, and realize the development of smart ocean virtual reality system step by step, by level and by elements; and complete the overall planning for the development of smart ocean virtual reality system when the project is completed.

(2) Study the application mode of virtual reality electronic map and collect ocean big data at the same time. Based on the existing systems such as remote sensing satellites, coastal ocean observatories, sea-based marine observation systems, seafloor observatories, and stereoscopic observation constitute the stereo observation systems used to perform ocean observations in different dimensions and different spatial-temporal scales, ocean data is collected to obtain model materials of system 3D modeling.

(3) The development of the marine virtual reality roaming system is mainly based on the overall arrangement of the preliminary work, from the 
humanized expression of the virtual reality map design, and the design and implementation of the marine virtual reality roaming system based on Unity3D.

\subsection{Marine Life Data Collection and Model Generation}

The field data collection methods of marine life including diving collection, fishing trawl collection, Deep - sea submersible acquisition and other collection methods. This system collects the marine life data through the network large data collection, using the high-speed transmission capability of the $5 \mathrm{G}$ network, the model data is input through the Internet and stored in the server. They are made into a marine life 3D model database for later use when using AR Foundation plug-in to make marine life dynamic demonstrations. The marine data collected by the network are shown in Figure 1 below.

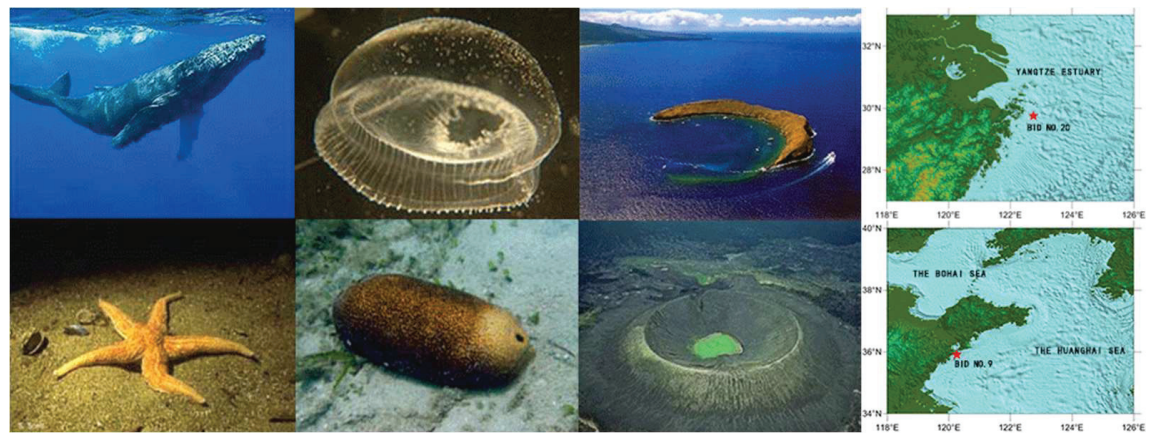

(Picture from https://www.sealifebase.se/search.php)

Figure 1 Large data collection of smart ocean system.

Under the influence of the COVID-19 in 2020, the system plans to add fresh seafood model, aiming to help the coastal import and export seafood traders identify whether the seafood comes from the infected area, and mark the source and freshness of the seafood in real time that the merchants needed to purchase, so that the merchants can buy at ease.

\subsection{Smart Ocean System Data Collection and Model Generation}

\subsubsection{Ocean system data collection and processing by python}

Global Ocean Observing System now seeks to coordinate observations around the global ocean for three critical themes: climate, operational services, and marine ecosystem health. To address these expanded requirements, 
new observations and data are clearly needed. Nowadays, network data collection usually includes four collection methods: open data source collection, crawler capture, sensor and log collection. This study chooses to use pythonbased web crawler technology to collect ocean big data, because web crawler technology is a program that can automatically get web information, and has obvious advanced nature in the application stage. It can search data from the Internet and achieve functions through three aspects: data acquisition, data processing and data storage.

In the process of obtaining ocean big data by the web crawler, the scheduler needs to ask the URL manager for information to determine whether there is any URL information to be crawled. If the prompt result is affirmative, the scheduler will get the first address to be crawled from the URL manager. In this way, the scheduler can download the web page information and upload it to the parser through the address information provided by the URL, and the parser will analyze whether there is any valuable information existed. The above process cycles infinitely, and can not stop until the corresponding conditions are met, so the quality of obtaining ocean big data information can be guaranteed.

\subsubsection{Smart Ocean System Scene Model Creation}

The key to achieving the best interaction and immersion of the Smart Ocean Extended Reality Visualization System is the creation of the scene model. The authenticity of the interaction of the entire system is closely related to the effect of model construction. The intelligent ocean extended reality scene built in this research has a vast sea area and numerous marine life. Therefore, using the ocean big data collected by Python crawler technology in the early stage, based on the characteristics of complexity, fluidity and unclear boundary of ocean data, the topography and landforms with low mobility and ocean current changes were all modeled. At the same time, the data of sea water temperature, sea water movement, marine disaster and marine resources are collected, and the changes of ocean information are marked in real time on the completed system model.

This research uses Google Earth's DEM contour maps to model the submarine terrain, the modeling method is to open Google map in SketchUp software, and grasp the geographical location to be modeled. Conceptual terrain can be stretched according to the grid with sandbox tools. For accurate terrain, you need to import the contour lines into SketchUp, and click Create according to contour lines in the sandbox tool to get the terrain that needs to be modeled. For other models, SketchUp software can be used for manual 
modeling, paste the texture image on each surface of the landscape, and adjust the mapping size to make the photo match the surface of the landscape.

For other models, SketchUp software can be used for manual modeling. The collected ocean scene photo data are put into SketchUp software to build the model of key scenes. Use modeling tools such as points, lines, surfaces, and bodies to model directly in SketchUp software, and the facade of the model is mapped to build a real scene model. Photoshop is used to process the collected photos of each elevation of the real scene. In SketchUp, open the material tool, paste the texture image on each face of the real scene model, and adjust the mapping size to make the texture of the photo match the surface of the real scene model.

Therefore, this research can use Unity3D to make a visualized extended reality smart ocean system based on the big ocean data collected by these networks.

\section{Design and Implementation of Smart Ocean Visualization System Based on Unity3D}

\subsection{The Overall Architecture of Smart Ocean Visualization System}

The design idea of this system development is based on the ocean data collected under the background of large data, and uses the extended reality technology as the means to make the ocean data information present smart and dynamic, enable humans to manage production and life in a more refined and dynamic way. It helps the government to manage the ocean, helps marine researchers obtain survey data more intuitively, helps the seafood import and export trade businesses to identify the source of seafood and the epidemic situation, realizes the globalization of smart ocean, realizes the human sea interconnection in time, and promotes the public's understanding of the ocean. The overall structure of this study is shown in Figure 2.

\subsection{System Implementation of Smart Ocean Visualization System}

\subsubsection{The overall construction of smart ocean XR scene}

After completing the fine modeling of the main ocean terrain and the rough modeling of the secondary scenes through SketchUp software, Unity3D software is needed to build the overall XR scene. First of all, we need to collect the basic information of the ocean scene, and establish the three-dimensional 


\section{Architecture of Smart Ocean Visualization System}

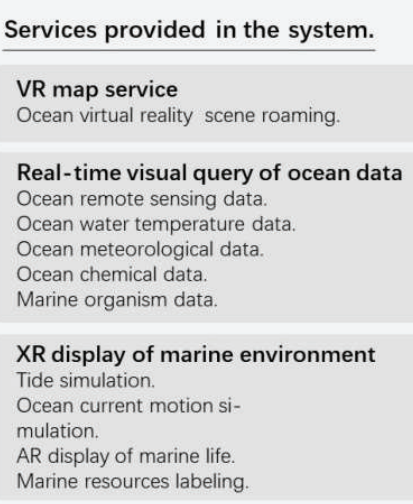

Operating functions of the system.

1.Color the selected ocean range to distinguish the selected area.

2.Toggle country (country adjacent ocean is displayed for selection) / frontier /label.

3.Change interface style (earth / border /color).

4.Quick positioning of ocean coordinates (located by ocean name, place name, and coordinate point).

5.Map manipulation (zoom, rotate, and move).

Figure 2 Architecture of smart ocean visualization system.

model of topography, materials and various ocean elements in the scene with accurate key dimensions and complete attribute parameters. Determine the structure size, shape, spatial position and other relations, and then under the modeling criteria, according to the data collected in the early stage (including planning maps, hand drawn planar graph and photos collected from the Internet), the created individual model is placed in SketchUp software according to the map position displayed in the data. Finally, import all 3D models into 3 dsmax software for baking and optimization, and export the FBX file model that unity $3 \mathrm{~d}$ software can support to the greatest extent to form an overall smart ocean XR scene.

\subsubsection{Realization of interactive function in smart ocean visualization system}

(1) Interactive Design Process

In the choice of virtual reality platform, this research chooses Unity3D software as the development platform of the smart XR system. We selected Unity3D, the most widely used game engine in the world, to build our proposed geospatial platform, because of Unity3D's advantages of convenience, accessibility, and extensibility of external libraries. Unity3D is capable of debugging various execution environments and is suitable for use in developing applications for various purposes. The scene is baked and rendered by Enlighten global real-time lighting technology and Lightmap baking 
technology to form a realistic ocean scene. In this study, the virtual scene includes real-time information query in the ocean scene, height adjustment in the scene, dynamic navigation setting of scene roaming and other related functions, and the interactive function realized by the interaction settings of unity3d software. The interaction modes include keyboard, mouse, event trigger, etc., and interact with various marine elements and marine life in the ocean scene.

\section{(2) Interactive Interface Effect}

In terms of the overall effect of the main interface, the system uses SketchUp software for manual modeling, so the effect of the main interface can fit the offline marine environment very well. Using the highly perfect lighting rendering system with soft shadows and lightmaps provided by unity $3 \mathrm{~d}$ software, and integrating with the leading real-time lighting technology Enlighten of Geomerics industry, the optimized real-time global lighting technology is realized in PC, host and mobile terminal. When the user opens the main interface, not only can they obtain the corresponding marine data information more conveniently, but also can be immersed in the virtual marine environment. As shown in Figure 3.

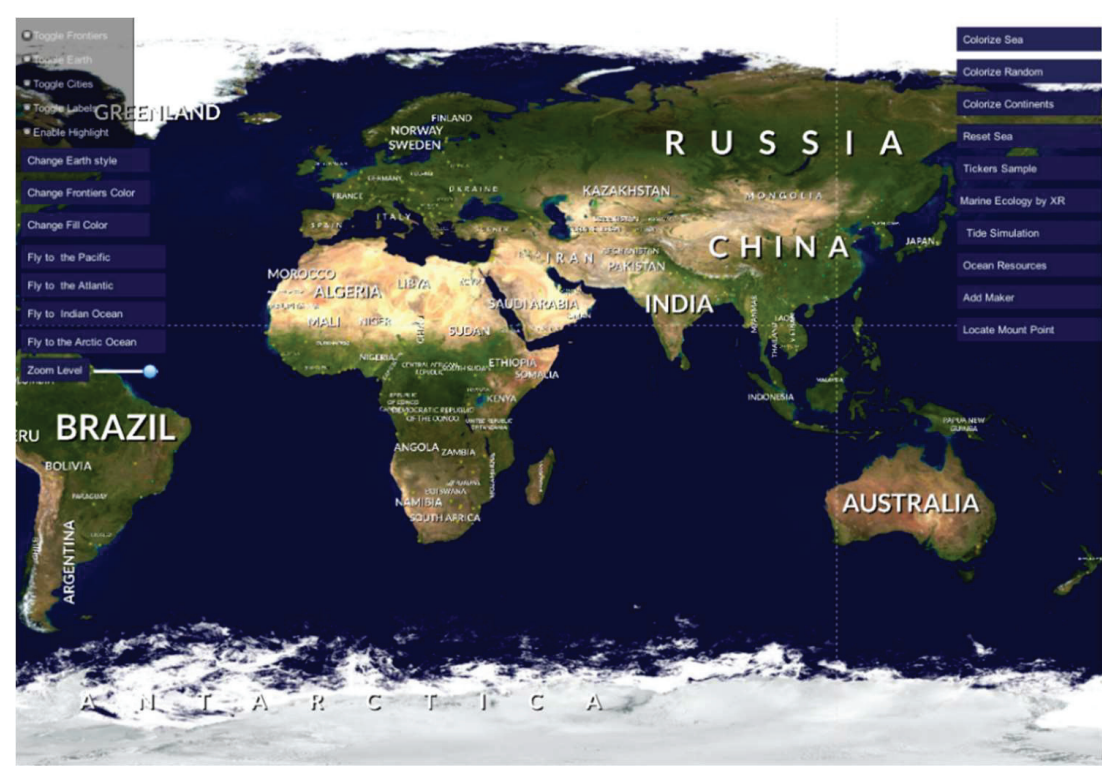

Figure 3 Effect drawing of main interface. 
In the system interface design, ocean blue is the main color, and ocean elements are used as UI design inspiration. The system interface colors, fonts and icons are designed, as shown in Figure 4 below.

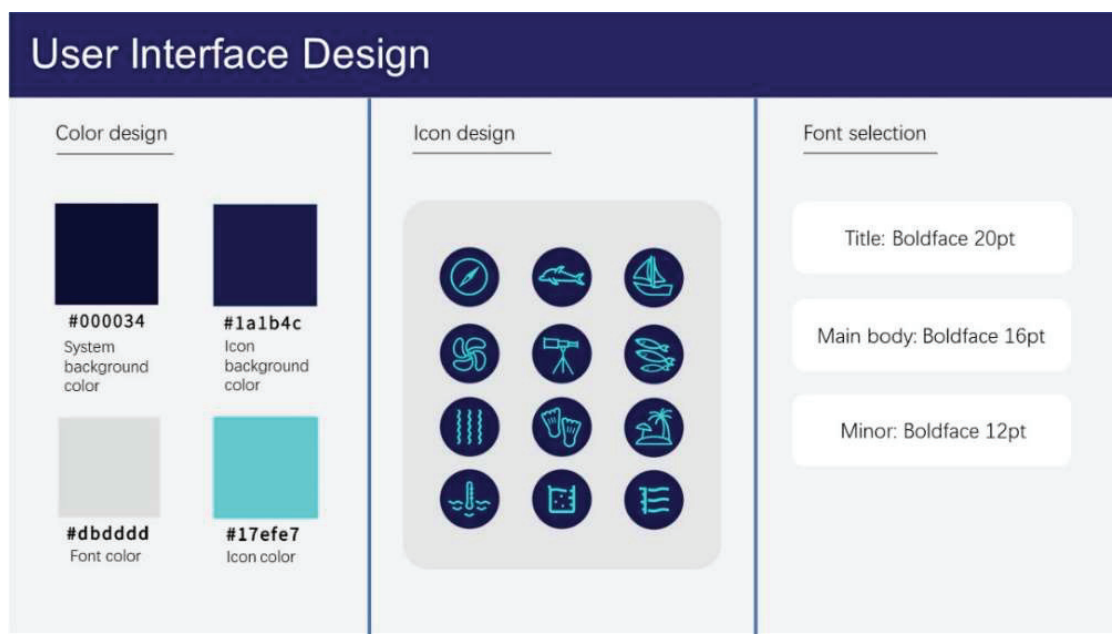

Figure 4 User interface design.

In the interface of the scene description, various ocean elements such as sea temperature, ocean current movement and marine life names have corresponding text descriptions. The content of the text description is usually distributed directly above the user interface. The gray canvas with a transparency of 50\% is used as the background color, and white is the word color. The contrast between the word color and the background color is obvious. See detailed instructions.

\section{(3) Interactive Implementation of XR}

In the realization of VR visualization interaction, through the built ocean scene, users can control the mobile terminal or computer perspective to move, realize the roaming in the ocean scene, and view the ocean information they need in VR while roaming. At the same time, it can also realize the VR visualization of ocean data, through the display of the VR data visualization chart in the smart ocean scene, and the mark of the VR information on the model in the scene, to realize the VR interaction of the smart ocean visualization system.

As for the realization of $\mathrm{AR}$ visualization and interaction, taking the biological model whale in the ocean scene as an example, the AR whale 
model made by AR foundation platform has the following steps: creating eclosion plane coloring device, creating unlit shader in shader; adding real shadows to add mobile AR shadow; modify the Lerp linear interpolation blending, sample through the given texture and coordinates, and mix the main texture and deformed color under AR through linear difference; the UI/UX framework under AR is constructed, and the two-step UI is used. The goal is to guide the users to find the plane. After finding the plane, the user can click to place the object, and after placing the object, the UI fades out.

The AR whale model produced by the AR Foundation platform can be combined with real water on the mobile interface, so that the real and virtual information can be supplemented and superimposed with each other through augmented reality technology, simulating the AR scene of whale swimming in the ocean, and seamlessly combining the movement of the whale with the real water body. As shown in Figure 5 below.

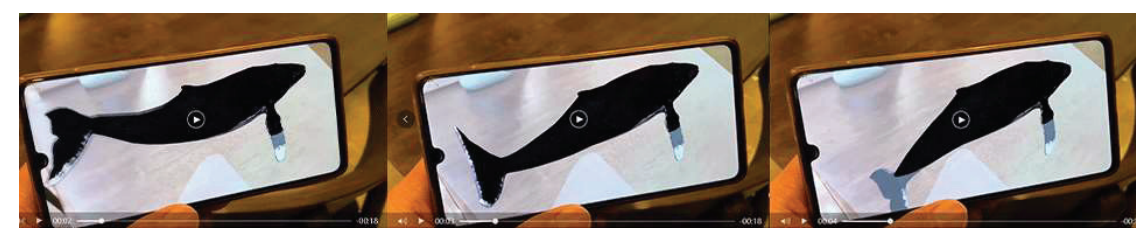

Figure 5 AR dynamic visual display of a whale.

In terms of the production of fish schools in the scene, the use of the Fish Flock plug-in enables the fish in the scene to move in groups and always remain as a group. They gather around the boundary of a hidden cube area, and avoid colliding with each other while swimming at the same time.

\section{(4) Interactive Operation Function}

In terms of user perspective control, when the user enters the system interface, he will roam in the scene from the first perspective. In the computer terminal, the code is set to control the speed of the character's movement and angle turning. In operation, the user controls the line of sight and movement mode of the characters in the system through the keyboard combined with mouse or finger touch, so as to capture the information in the scene required by the user.

\subsection{Export of Smart Ocean Visualization System}

After completing the construction of the smart ocean system in the unity software, export the installation package of the system. The first step is 
to create a new project Unity_Build_To_Android. The second step is to create the following four files. First, to demonstrate the native Android jar package, Plugins/Android/libs/Jar.jar. The other is to demonstrate the .so file needed by native Android to call jni, Plugins/Android/libs/x86/libnative.so. Third, to demonstrate the so file needed by native Android to call jni, Plugins/Android/libs/armeabi-v7a/libnative.so. Four is for Demonstrate the direction of read-only files with packages in unity, StreamingAssets/ALL_EmptyTxt.txt. The third step is to save the scene. The fourth step is to open the Player Settings and modify the Bundle Identifier (package name). The fifth step is to select the Android platform and export to export the Android project. The sixth step is to use AndroidStudio to open the Eclipse Project, select Unity to export the Eclipse project, and store it to the appropriate path. After setting, the installation package of smart ocean system can be released.

\section{Summary and Outlook}

\subsection{Project Innovation}

The most important innovation of this research is the use of extended reality technology to innovate the two-dimensional visualization of traditional application interfaces. It breaks through the plane presentation of traditional interfaces on mobile terminals. Through the combination of virtual reality and augmented reality, it displays ocean scenes and images of marine life in a three-dimensional visualization way. The specific innovations are as follows:

\subsubsection{Smart data query}

The work of marine researchers includes marine hydrological observation, marine pollution monitoring and sea area investigation. In marine hydrological observation, it is necessary to observe the changes of water depth, water temperature, salinity, current, wave, water color, transparency, sea ice, sea light and other marine hydrological elements on time. In this system, researchers can mark the changes of marine hydrological elements observed by the observation station in the smart ocean XR system in real time, which makes it possible to understand the distribution and laws of hydrological elements in an intuitive and visual way. At the same time, the marking of marine data information in the system can let passing ships know the route status in advance and reduce the navigation risk. At the same time, in view of the impact of the current epidemic situation of the COVID-19, the VR model 
of seafood is added into the system to mark the source data and infection information data of seafood in real time, so that the purchasing merchants can buy at ease and the consumers can eat at ease.

\subsubsection{Smart ocean tourism}

The online virtual reality ocean scene roaming allows tourists to see $100 \%$ of the highly realistic three-dimensional scenes such as islands and diving places recommended for travel in the sea area, and customize the travel strategy in advance. Meanwhile, the marine landscape explanation, intelligent route recommendation and edible seafood introduction built into the XR system will be accompanied during the journey, so that tourists can get a deeper ocean life experience they don't know on land in marine tourism. In addition, functions such as online transactions can be developed in the future, taking advantages of $5 \mathrm{G}$ era and the rapid development of fresh logistics to allow local seafood merchants to enter the XR system, so that mainland tourists can still see the realistic three-dimensional seasonal seafood after the trip, and can be purchased from their favorite local merchants.

\subsection{Summary and Outlook}

In conclusion, this project is designed to solve the problems of visualization of marine data information and the development of Marine Tourism under the background of building a maritime power. Using XR technology to create an extended reality smart ocean system through the Unity3D platform, the innovation of three-dimensional visualization data query methods is realized in ocean data query, and at the same time, the three-dimensional presentation of highly realistic ocean scenes helps the development of ocean tourism.

For the government, China has a vast sea area under its jurisdiction and abundant marine resources. At present, the development and utilization of marine resources are generally not high. China basically has the resources, environment and geographical conditions to move towards the sea, and the marine resources have great potential to support the country's social and economic development. The system is one of the means to help the government effectively realize the construction of a maritime power. The development of the smart ocean system can make the ocean management three-dimensional and intelligent, present the marine data in a centralized and visual way, get rid of the fragmented two-dimensional representation of ocean data in the past, and improve the work efficiency of the government on ocean management.

For marine researchers, this project can make it easy and intuitive to collect ocean data from the XR system during the research process. Compared 
with the previous two-dimensional fragmented ocean data information, this system can make the researchers obtain the centralized and open data information more easily, so that they can pay more attention on the research work, instead of wasting their time on searching fragmented ocean data.

For the general public, the development of online XR ocean system, on the one hand, for the marine product traders, the seafood is visualized in the form of XR, and includes the source and virus pollution information annotations, which can make customers more intuitive to see the seafood and buy at ease. On the other hand, for tourists, it allows tourists to see the true appearance of ocean attractions more clearly, recognize and understand the mysterious ocean that is difficult to reach on land, stimulate tourists' interest in the ocean, and learn about information needed to know of ocean tourism in advance, so as to promote the desire of marine tourism from virtual to real, and then push forward the development of marine tourism.

In the visualization of marine data information, the extended reality system of Smart Ocean can bring users a more convenient experience and a more immersive using experience than traditional two-dimensional data systems. It is a supplement and improvement to the traditional two-dimensional marine data system. In tourism, in the 5G era, the Ocean Extended Reality System allows users to have an immersive travel experience through time and space. The development of smart ocean extended reality visualization system, in the continuous feedback modification and improvement, can be subsequently supplemented with data from various sea areas around the world, creating a smart and interconnected world ocean extended reality system for a more convenient management of the government, a faster inquiry speed for the scientific research personnel to search ocean data, and promoting the development of Coastal businesses and the willing of marine tourism of the public.

\section{Acknowledgements}

This work is supported by Youth Foundation of Social Science and Humanity, China Ministry of Education (Grants No. 20YJC760025), the scientific research project of Hubei Provincial Department of Education (Grants No. q20191402), the Funding of The Innovation and Entrepreneurship Program for College Students of the Ministry of Education of China (Grants No. 201810500024), the Key Projects of the Open Fund of the Hubei Cultural and Creative Industrialization Design and Research Center (Grants No. HBCY201901), the Funding of The Innovation and Entrepreneurship 
Program for College Students of the Education department of Hubei province, China (Grants No. 201910500044), the Key Projects of the campus culture cultivation Fund of the Hubei University of Technology (Grants No. 2019SW0109), and the Innovation and the green technology leadership program of the Hubei university of technology (Grants No. CPYF2018009), Fund of Hubei University of Technology (Grants No. BSQD2017050).

\section{References}

[1] X. Y. Jiang, and D. L. Pan. 2018. Suggestions on the development of the Smart Ocean in China. Marine Information, (1), 1-6.

[2] Z. W. Li, J. Fu, and H. X. Han. 2019. Design and Implementation of Three-dimensional Visualization System for Marine Environmental Data. Hydrographic Surveying and Charting, 39(06), 68-72.

[3] E. Havice, M. Marschke, and P. Vandergeest. 2020. Industrial seafood systems in the immobilizing COVID-19 moment. 2020, 37(prepublish):1-2.

[4] H. J. Zhao, Z. L. Yang, H. X. Liu, Y. K. Chen, W. Wang, L. K. Kuang, and X. Y. Zhu. 2020. Impact of novel coronavirus pneumonia on China's import and export aquatic products industry and countermeasures. Journal of Food Safety \& Quality, 11(22), 8180-8185.

[5] Škare Marinko, Soriano Domingo Riberio, Porada Rochoń Małgorzata. 2020. Impact of COVID-19 on the Travel and Tourism Industry. 2020: 120469-.

[6] S. W. Zhang. 2020. Decoding data and drawing the sea: record Marine Artificial Intelligence and Large Data Center. Science News, (04), 72 73.

[7] Keating Timothy C, and Jacobs Joshua J. 2020. Augmented Reality in Orthopedic Practice and Education. 2021, 52(1):15-26.

[8] E. G. İsmailoğlu and A. Zaybak. 2020. Comparison of the effectiveness of a virtual simulator with a plastic arm model in teaching intravenous catheter insertion skills, CIN 36(2) (2018), 98-105.

[9] J. H. Yin, C. B. Chng, P. M. Wong, et al. 2020. VR and AR in human performance research - an NUS experience. 2020, 2(5):381-393.

[10] Moreira, M. C., Am, D. A. L., Ferraz, K. M., \& Benedetti Rodrigues, M. A. 2013. Use of virtual reality in gait recovery among post stroke patients - a systematic literature review. Disability \& Rehabilitation Assistive Technology, 8(5), 357. 
[11] Y. Zhong. 2020. Research on Ocean Data Big Data Management Technology. Computer products and circulation (in Chinese), (02), 145.

[12] X. Song, Y. Guo, Y. Chang, F. Zhang, J. Tan, J. Yang, X. Shi. 2020. A Hybrid Recommendation System for Marine Science Observation Data Based on Content and Literature Filtering. Sensors (Basel). 2020. Nov 10;20(22):6414.

[13] Y. Gu, and X. M. Qi. 2020. Construction of Agricultural Big Data Visualization Platform Based on GIS in Nanjing City. Journal of Shandong Agricultural University (Natural Science Edition), 51(4), 1-3.

[14] T. Moltmann. J. Turton and H. M. Zhang, et al. 2019. A Global Ocean Observing System (GOOS), Delivered Through Enhanced Collaboration Across Regions, Communities, and New Technologies. Frontiers in Marine Science, 2019, 6.

[15] Q. Z. Pan and L. Zhang. 2018. Discussion on python-based web crawler technology in big data environment. Network Security Technology \& Application, (05), 41-42.

[16] Z. Q. Li, Z. H. Liu, J. P. Xu, C. H. Sun, and S. L. Lu. 2020. The Multiplatform Data Management Specification for 'Smart Ocean'. Ocean Development and Management, 37(04), 78-83.

[17] S. Wang, Z. Mao, C. Zeng, H. Gong, S. Li, B. Chen. 2010. A new method of virtual reality based on Unity3D. In Proceedings of the IEEE International Conference on Geoinformatics, Beijing, China, 18-20 June 2010; pp. $1-5$.

[18] J. Xie. 2012. Research on key technologies base Unity3D game engine. In Proceedings of the IEEE International Conference on Computer Science \& Education (ICCSE), Melbourne, Australia, 14-17 July 2012; pp. 695-699.

[19] Koh, E., Park, G., Lee, B., Kim, D., Sung, S. 2020. Performance Validation and Comparison of range/INS integrated system in urban navigation environment using Unity3D and PILS. In Proceedings of the 2020 IEEE/ION Position, Location and Navigation Symposium (PLANS), Portland, OR, USA, 20-23 April 2020; pp. 788-792.

[20] J. Q. Zhou, We. H. Chen, D. Li, and Z. H. Yang. 2020. Design and Implementation of Nuclear Power Somatosensory Roaming System Based on Unity3d and Kinect. Electronic Instrumentation Customers, 27(06), 39-44. 
[21] Y. Z. Ma, and W. Sang. 2020. Implementation of An Augmented Reality Platform to Support Operation and Maintenance in Nuclear Power Plants. Electronic Instrumentation Customers, 27(09), 62-65.

[22] F. Wang. 2020. Research on the Situation and Tasks of Building a Strong Maritime Power in the New Era. Journal of Ocean University of China (Social Sciences), (05), 11-19.

\section{Biographies}

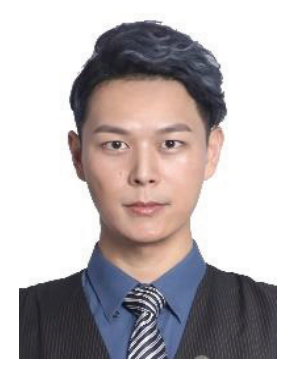

Xu Han, was born in 1987 in Wuhan, Hubei Province, China. He graduated from Wuhan University in 2016 with a doctorate degree in engineering. Since 2017, he has been a lecturer in the Department of Interaction Design at the School of Industrial Design, Hubei University of Technology. His research interests include virtual reality, human-computer interaction, and landscape design.

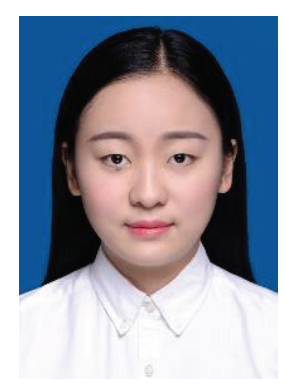

Liu Jingming is a postgraduate student at the Hubei University of Technology since autumn 2018, and major in Industrial Design Engineering. She attended the Guilin University of Technology where she received her 


\section{X. Han et al.}

B.Sc. in Logistics Management in 2017. Her postgraduate work centers on virtual reality and interaction design.

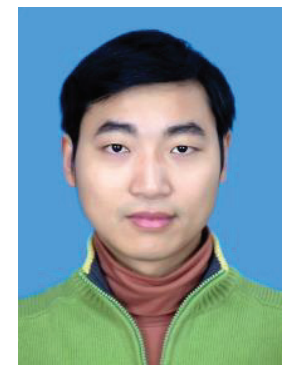

Baohua Tan, was born in Yingshan, Hubei Province, China in 1978. He received the Ph.D. degree in Power Machinery and Engineering from the Wuhan University, of Technology, Wuhan, in 2014. Since 2016, he has been a Professor of the School of Science, the Hubei University of Technology, Wuhan, China. His research interests include the information exchange technology, computer application technology, measurement and control technology, and photovoltaic technology.

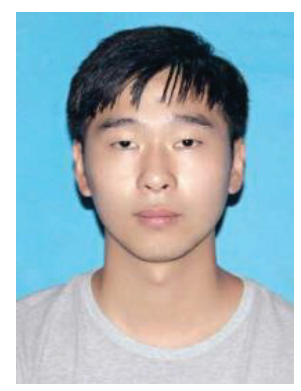

Duan Lucheng is a master student at the Hubei University of Technology since September,2020. He got his bachelor's degree from the school of computer science of Hubei University of Technology. And now he is studying for a master's degree in integrated circuit. He is good at data analysis, embedded software programming and circuit analysis. 